ORIGINAL ARTICLE

\title{
Glutathione S-transferases $M 1-1$ and $11-1$ as risk modifiers for renal cell cancer associated with occupational exposure to chemicals
}

\author{
L Buzio, G De Palma, P Mozzoni, M Tondel, C Buzio, I Franchini, O Axelson, A Mutti
}

See end of article for

Occup Environ Med 2003;60:789-793

authors' affiliations

Correspondence to:

Professor A Mutti

Laboratory of Industrial

Toxicology, Dept of

Clinical Medicine,

Nephrology and Health

Sciences, University of

Parma, via Gramsci 14,

43100 Parma, Italy;

antonio.mutti@unipr.it

Accepted

24 November 2002

\begin{abstract}
Aims: To investigate the possible interaction between occupational risk factors and genotype for glutathione S-transferases M1 and T1 (GSTM1 and GSTT1) in renal cell cancer (RCC).

Methods: One hundred patients with RCC and 200 outpatient controls were enrolled at Parma University Hospital. The polymorphisms of glutathione S-transferase M1-1 (GSTM1) and T1-1 (GSTT1) were investigated by PCR; occupational history was collected by a structured questionnaire.

Results: Subjects with GSTM1 present genotype showed higher risks for RCC, compared to GSTM1 null subjects, if exposed to metals (OR 2.73; $95 \% \mathrm{Cl} 0.91$ to 8.22 v $1.14 ; 95 \% \mathrm{Cl} 0.46$ to 2.82 ) or pesticides (OR $3.46 ; 95 \% \mathrm{Cl} 1.12$ to 10.74 v $1.59 ; 95 \% \mathrm{Cl} 0.48$ to 5.34 ). The GSTT1 present genotype also enhanced the risk (about twofold) of RCC among subjects exposed to solvents and pesticides, compared with those GSTT1 null.

Conclusions: Results support the hypothesis that GSTM1 and GSTT1 polymorphisms can interact with several occupational exposures to significantly modify the risk of RCC among exposed subjects.
\end{abstract}

$\mathrm{R}$ enal cell cancer (RCC) represents about 3\% of all tumours in adults, with increasing incidence in Europe, ${ }^{1}$ as well as in the United States. ${ }^{2}$ The aetiology of RCC remains unclear, though epidemiological studies indicate that therapeutic agents, ${ }^{3}$ smoking habits, ${ }^{4}$ obesity, ${ }^{5}$ and occupational exposure to chemicals ${ }^{6}$ may play a role in the development of this tumour. Occupational exposures to organic solvents, metals, and pesticides have been associated with RCC. ${ }^{78}$ Mutations of the Von Hippel-Lindau (VHL) tumour suppressor gene, known to occur in $55-70 \%$ of familial cases, ${ }^{9}$ showed an increased prevalence among RCC cases with a positive history of prolonged occupational exposure to high levels of trichloroethylene (TRI), ${ }^{10}$ indicating that a gene-environment interaction may underlie RCC. Other genes have been implicated in the pathogenesis of RCC, such as the tuberous sclerosis gene 2 (TS2) ${ }^{11}$ and the WTl gene, which interfere with several oncogenes, such as bcl-2 and c-myc. ${ }^{12}$ Soluble glutathione S-transferases (GST) represent a superfamily of inducible enzymes, comprising at least seven classes of cytoplasmic proteins $(\alpha, \mu, \pi, \sigma, \theta, \kappa, \zeta),{ }^{13}$ which catalyse the conjugation of glutathione (GSH) with different species of electrophilic compounds. As an effect of genetic polymorphism, GSTMl and GSTTl activity are lacking in about $50 \%$ and $20 \%$, respectively, of Caucasians because of homozygous deletions of the respective genes (GSTMI and GSTTI). ${ }^{14}$ The corresponding genotypes are defined as GSTMI null and GSTT1 null, whereas subjects retaining at least one wild-type allele, and hence displaying GSTMI-1 and/or GSTT1-1 activity, are indicated as bearing a GSTM1 present and/or a GSTTI present genotype, respectively. Inconsistent results have been reported by studies investigating the role of GSTMI and GSTT1 as risk factors for RCC. ${ }^{15} 16$ A significantly higher prevalence of GSTM1 present and GSTT1 present genotypes has been shown by Brüning et al among sporadic RCC cases with a history of heavy exposure to TRI compared to unaffected and similarly exposed subjects. ${ }^{17}$

The aim of the present case-control study was to investigate the role of the GSTMI and GSTT1 polymorphisms as risk modifiers of RCC associated with occupational exposure to chemicals.

\section{METHODS}

\section{Recruitment of subjects}

One hundred patients (62 males), with histologically confirmed diagnosis of RCC, were enrolled from the day hospital of the Department of Clinical Medicine, Nephrology and Health Sciences at Parma University, when attending for periodic checks. The control group included 200 patients (120 males) with non-neoplastic diseases, randomly selected from outpatient specialist centres of the same university hospital. Of these 200 controls, 107 patients were attending the haemostasis centre; of the remaining controls, 52 came from the nephrology, 27 from the cardiology, 11 from the ophthalmology, and 3 from the dermatology clinics. Diagnoses were vascular disease (receiving anticoagulant treatment), renal hypertension or chronic renal failure, essential hypertension, glaucoma, and psoriasis. The participation rate differed to some extent depending on the recruiting outpatient centre but was, on average, $90 \%$ among controls compared with $97 \%$ among cases. The study protocol was approved by the local ethics committee and all subjects gave their written and informed consent. The mean age was 63.1 (SD 8.9) years for interviewed cases and 68.8 (SD 9.5) years for the control group. The prevalence of smokers and ex-smokers was higher among controls than cases $(59.5 \%$ versus 54\%). Both such percentages were higher than those of the general population in Italy, which at the time of the interviews was $46 \%$. There was no difference in gender between cases and controls ( $p=0.87, \chi^{2}$ test), and also when the sample was stratified for classes of age (table 1).

\footnotetext{
Abbreviations: GSH, glutathione; GST, glutathione S-transferase; OR, odds ratio; PCR, polymerase chain reaction; RCC, renal cell cancer; TRI, trichloroethylene
} 


\section{Main messages}

- In gene-environment interactions, polymorphic xenobiotic metabolising enzymes may play a role as risk modifiers, such a role being only apparent in subjects exposed to relevant substrates.

\section{Exposure assessment}

Cases and controls were interviewed using a structured questionnaire identifying sociodemographic variables, including smoking habits and occupational and medical histories. Information on occupational titles held for more than one year, type of industry during working life, and the number of years of exposure to chemicals or raw materials was collected. Exposure to chemicals at the workplace was assessed blindly by an industrial hygienist unaware of the case/control status. The exposed group comprised subjects occupationally exposed for one year, or more, to at least one of the categories of agents under study (organic solvents, metals, and pesticides).

\section{Genotyping}

The genotypes of GSTMI and GSTT1 were characterised on DNA extracted from $5 \mathrm{ml}$ of peripheral venous whole blood by the Nucleon BACC2 commercial kit (Amersham Life Science, Little Chalfont, UK). Genetic polymorphisms were determined, according to published methods, by a multiplex polymerase chain reaction (PCR), using albumin as the internal control. ${ }^{18}$ Among controls, the observed genotype and genotype combination frequencies, reported in table 2 , were not significantly different from frequencies previously described among Caucasians. ${ }^{14}$

\section{Statistical analysis}

Logistic regression was used to estimate the odds ratio (OR) along with $95 \%$ confidence intervals (95\% CI). ${ }^{19}$ Significantly increased ORs were referred to when the lower confidence interval exceeded 1.00. All ORs presented here were adjusted by age and smoking habits (current and ex-smokers versus subjects who had never been smokers). In the analysis of gene-environment interaction, we used non-exposed subjects (that is, those not exposed to solvents, metals, and pesticides) with null genotype as the reference. When the combinations of GSTMI and GSTTl genotypes were considered together with past exposures, none of the cases belonged to the category of GST null people with no exposure. Therefore, the reference group was composed of unexposed subjects bearing either GSTMl null or GSTT1 null. The $p$ value was regarded as significant if it did not exceed 0.05 . All analyses were performed using the statistical package SPSS for Windows (version 10.0). Statistical power for gene-environmental interaction was calculated using the software program QUANTO. ${ }^{20}$

\section{Policy implications}

- GST positive individuals might require special protection against the risk of renal cancer associated with exposure to metals, solvents, and pesticides.

- Further studies on much larger populations should be carried out to confirm these findings and to identify specific agents playing a causal role in renal cell cancer.

\begin{tabular}{|c|c|c|c|c|}
\hline & \multicolumn{2}{|l|}{ Cases } & \multicolumn{2}{|c|}{ Controls } \\
\hline & Male & Female & Male & Female \\
\hline \multicolumn{5}{|l|}{ Age (years) } \\
\hline$\leqslant 50$ & 6 & 4 & 12 & 7 \\
\hline$>50 \leqslant 60$ & 14 & 11 & 31 & 27 \\
\hline$>60 \leqslant 70$ & 30 & 13 & 46 & 26 \\
\hline$>70 \leqslant 80$ & 11 & 9 & 28 & 16 \\
\hline$>80$ & 1 & 1 & 3 & 4 \\
\hline \multicolumn{5}{|c|}{ Smoking habits (males and females combined) } \\
\hline Current & \multicolumn{2}{|l|}{29} & \multicolumn{2}{|l|}{56} \\
\hline Ex-smokers & \multicolumn{2}{|l|}{25} & \multirow{2}{*}{\multicolumn{2}{|c|}{63}} \\
\hline Never smokers & & & \\
\hline $\begin{array}{l}\text { Pack-years, mean } \\
\text { (SE) }\end{array}$ & \multicolumn{2}{|c|}{$33.28(3.55)$} & \multicolumn{2}{|c|}{$30.21(2.47)$} \\
\hline
\end{tabular}

\section{RESULTS}

In our series, GSTMl present genotypes were over-represented among cases compared to GSTMI null (OR 1.19; 95\% CI 0.73 to 1.92), as were GSTT1 present versus GSTTI null (OR 1.72; $95 \%$ CI 0.83 to 3.55$)$, but without reaching statistical significance (table 2). Among cases, compared to controls, the combination of GSTMI present and GSTT1 present genotype prevailed over the combination including at least one defective genotype, but the difference was not statistically significant. Table 3 shows the influence of the GSTMI polymorphism on the risk for RCC, among occupationally exposed subjects. The GSTMI present genotype was associated with an increased risk for subjects exposed to metals (OR $2.73 ; 95 \%$ CI 0.91 to 8.22 ) or pesticides (OR 3.46; 95\% CI 1.12-10.74)(OR 2.73; 95\% CI 0.91 to 8.22 ) or pesticides (OR 3.46; $95 \%$ CI 1.12 to 10.74); whereas the same exposures gave lower point estimates for the risk among GSTMI null subjects (OR of $1.14 ; 95 \%$ CI 0.46 to 2.82 , and 1.59 ; $95 \%$ CI 0.48 to 5.34 , respectively). The distribution of GSTMI present and GSTMI null genotypes among cases and controls exposed to solvents was not remarkably different. The GSTT1 genotype status played a significant modulating role in the association between solvent exposure and RCC (table 4); the odds ratio for GSTT1 present subjects was twofold greater than in GSTTI null subjects (OR 7.23; 95\%

Table 2 Odds ratios (OR) for renal cell cancer and their association with glutathione S-transferases M1-1 and T1-1 (GSTM1 and GSTT1) as adjusted for age and smoking habits

\begin{tabular}{llll}
\hline Genotypes & Cases & Controls & OR (95\% Cl) \\
\hline GSTM1 present $v$ GSTM1 null & $50 / 50$ & $92 / 108$ & 1.19 (0.73 to 1.92) \\
GSTT1 present $v$ GSTT1 null & $89 / 11$ & $165 / 35$ & 1.72 (0.83 to 3.55) \\
Genotype combinations & $45 / 55$ & $73 / 127$ & 1.40 (0.85 to 2.29) \\
GSTM1 present and GSTI1 present $v$ else* & 45 \\
\hline
\end{tabular}

*At least one GSTM1 or GSTI1 defective genotype is included in the combination. 
Table 3 Odds ratios (OR) for renal cell cancer in relation to occupational risk factors as adjusted for age and smoking habits, and stratified for glutathione S-transferase MI-1 (GSTM1)

\begin{tabular}{|c|c|c|c|c|}
\hline Genotypes & Cases* & Controls* & OR $(95 \% \mathrm{Cl})$ & $p$ for interaction \\
\hline \multicolumn{5}{|l|}{ No exposure } \\
\hline GSTM1 null & 21 & 63 & 1.00 (reference) & \\
\hline GSTMI present & 22 & 66 & $0.98(0.48$ to 1.96$)$ & \\
\hline \multicolumn{5}{|c|}{ Exposure to solvents } \\
\hline GSTM1 null & 15 & 15 & 3.06 (1.26 to 7.43$)$ & \\
\hline GSTM1 present & 11 & 13 & $2.23(0.82$ to 6.12$)$ & 0.572 \\
\hline \multicolumn{5}{|c|}{ Exposure to metals } \\
\hline GSTM1 null & 10 & 27 & 1.14 (0.46 to 2.82$)$ & \\
\hline GSTM1 present & 9 & 9 & $2.73(0.91$ to 8.22$)$ & 0.093 \\
\hline \multicolumn{5}{|c|}{ Exposure to pesticides } \\
\hline GSTM1 null & 11 & 19 & $1.59(0.48$ to 5.34$)$ & \\
\hline GSTM1 present & 15 & 11 & 3.46 (1.12 to 10.74$)$ & 0.445 \\
\hline
\end{tabular}

Subjects with GSTM1 null and none of the exposures under study were used as reference. *Twelve cases and 20 controls with double exposure.

CI 1.87 to $27.87 v 3.12 ; 95 \%$ CI 0.38 to 25.48$)$. The difference between the point estimates of the risk for GSTT1 present and GSTT1 null individuals exposed to metals was rather strong (OR 3.93; 95\% CI 1.00 to 15.45 , and 1.22; $95 \%$ CI 0.08 to 18.10, respectively). Among subjects with a past exposure to pesticides, higher ORs were found in GSTT1 present groups (OR 6.54; 95\% CI 1.49 to 28.81 , and $4.37 ; 95 \%$ CI 0.46 to 41.57 , for GSTT1 present and GSTT1 null, respectively). When the combinations of GSTM1 and GSTT1 genotypes were considered together with past exposures (table 5), we observed an absence of cases among those with no exposure, without taking the genotype into account. GSTM1 present and GSTT1 present subjects were significantly over-represented among exposed RCC cases, with OR values ranging from 2.91 (95\% CI 1.06 to 7.99 ) for exposure to solvents, to 6.64 (95\% CI 1.81 to 24.45) for exposure to pesticides. OR values of the GSTMI present and GSTT1 present subgroup exceeded those found for other genotype combinations in any exposure group.

\section{DISCUSSION}

Whereas a previous analysis of the same sample focused on environmental and occupational factors, ${ }^{21}$ in the present case-control study we considered gene-environment interactions associated with RCC. In particular, the modifying role of polymorphic GSTMI and GSTTI on the risk of RCC among subjects occupationally exposed to different chemicals was investigated. Only two general population based studies are available on the risk of RCC in relation to GSTM1/GSTT1 status, and the results seem to be inconsistent. In a French case-control study, GSTMI present and GSTT1 present genotypes were slightly, and non-significantly, over-represented among RCC patients. ${ }^{15}$ On the contrary, an American case-control study suggested an association between RCC and the GSTT1 null genotype, with an OR of 1.9 (95\% CI 1.1 to 3.4). ${ }^{16}$ Both studies analysed the association of GSTs with RCC, thus treating the genotype as a potential risk factor, rather than a potential risk modifier. However, it is difficult to conceive an association between polymorphic enzymes and any outcome, in the absence of exposure to the relevant substrate(s).

We chose to recruit cases and controls from outpatient clinics of the same university hospital in order to control for recall bias and to obtain a geographical balance between the two groups, thus creating equal possibilities for occupationalenvironmental exposure among cases and controls. Without considering previous occupational history, the GSTMI and GSTT1 genotypes in our own material are distributed with

Table 4 Odds ratios (OR) for renal cell cancer in relation to occupational risk factors as adjusted for age and smoking habits, and stratified for glutathione S-transferase T1 (GSTT1) activity

\begin{tabular}{|c|c|c|c|c|}
\hline Genotypes & Cases* $^{*}$ & Controls* & OR $(95 \% \mathrm{Cl})$ & p for interaction \\
\hline \multicolumn{5}{|l|}{ No exposure } \\
\hline GSTTl null & 3 & 21 & 1 (reference) & \\
\hline GSTTl present & 40 & 108 & 2.59 (0.73 to 9.17$)$ & \\
\hline \multicolumn{5}{|c|}{ Exposure to solvents } \\
\hline GSTT1 null & 2 & 5 & $3.12(0.38$ to 25.48$)$ & \\
\hline GSTTl present & 24 & 23 & 7.23 (1.87 to 27.87 ) & 0.585 \\
\hline \multicolumn{5}{|c|}{ Exposure to metals } \\
\hline GSTT1 null & 1 & 5 & $1.22(0.08$ to 18.10$)$ & \\
\hline GSTT1 present & 18 & 31 & 3.93 (1.00 to 15.45$)$ & 0.616 \\
\hline \multicolumn{5}{|c|}{ Exposure to pesticides } \\
\hline GSTT1 null & 6 & 6 & $4.37(0.46$ to 41.57$)$ & \\
\hline GSTT1 present & 20 & 24 & $6.54(1.49$ to 28.81$)$ & 0.919 \\
\hline
\end{tabular}

Subjects with GSTT1 null and none of the exposures under study were used as reference *Twelve cases and 20 controls with double exposure. 


\begin{tabular}{|c|c|c|c|c|}
\hline Genotype combinations & Cases* $^{*}$ & Controls* & OR $(95 \% \mathrm{Cl})$ & $\begin{array}{l}p \text { for } \\
\text { interaction }\end{array}$ \\
\hline \multicolumn{5}{|l|}{ Not exposed } \\
\hline Either GSTM1 null or GSTI null & 24 & 75 & 1.00 (reference) & \\
\hline GSTM1 present and GSTT1 present & 19 & 54 & $1.08(0.53$ to 2.18$)$ & \\
\hline \multicolumn{5}{|l|}{ Exposed to solvents } \\
\hline Either GSTM1 null or GSTT1 null & 15 & 18 & 2.65 (1.15 to 6.10$)$ & \\
\hline GSTM1 present and GSTT1 present & 11 & 10 & 2.91 (1.06 to 7.99$)$ & 0.152 \\
\hline \multicolumn{5}{|l|}{ Exposed to metals } \\
\hline Either GSTM1 null or GSTT1 null & 10 & 28 & $1.08(0.45$ to 2.60$)$ & \\
\hline GSTM1 present and GSTT1 present & 9 & 8 & $2.97(1.00$ to 8.89$)$ & 0.456 \\
\hline \multicolumn{5}{|l|}{ Exposed to pesticides } \\
\hline Either GSTM1 null or GSTT1 null & 13 & 24 & 1.21 (0.38 to 3.85$)$ & \\
\hline GSTM1 present and GSTT1 present & 13 & 6 & $6.64(1.81$ to 24.45$)$ & 0.016 \\
\hline
\end{tabular}

Non-exposed subjects bearing either GSTM1 null or GSTT1 null were used as reference.

*Twelve cases and 20 controls with double exposure.

frequencies that resemble the frequencies reported in the French study. In the group of non-exposed subjects GSTMI polymorphism distributes symmetrically, whereas GSTTl present is more represented among cases than controls (OR 2.59; CI $95 \% 0.73$ to 9.17 ), probably implying an exposure to endogenous or exogenous carcinogens not investigated by the questionnaire. When the role of GSTMI and GSTTI as risk modifiers for RCC was assessed, including previous occupational exposures, the GSTMI present and GSTTI present genotypes, either individually or combined, were associated with higher risks of developing RCC. The biological plausibility of these findings would involve a selective vulnerability of the tubular renal epithelium to toxic effects exerted by metabolites derived from GSH conjugated intermediates. Generally, the products catalysed by GSTs have higher solubility than the parent metabolite, making their excretion in urine or bile easier, after subsequent conversion into mercapturic acids. Depending on the chemical properties of the parent compound undergoing GSH conjugation, either toxic or mutagenic metabolites can be generated in organs and tissues expressing peptidases of the mercapturic acids pathway and $\beta$-lyases, for example, the liver and the kidney. ${ }^{22}$ A biological model has been proposed by several authors to explain the selective nephrotoxicity and nephrocarcinogenicity of halogenated solvents, particularly with respect to TRI. This compound is GSH conjugated to S-(dichlorovinyl)-GSH which, in the kidney and the biliary ducts, enters the mercapturic acid pathway, being cleaved to S-(1,2-dichlorovinyl)-L-cysteine. In the renal tubular epithelium, this metabolite is enriched and can be bioactivated by cysteine conjugate $\beta$-lyases to highly reactive chlorothioketenes, which can form adducts with proteins and DNA. ${ }^{23}$ An increased incidence of RCC was reported among workers exposed to high doses of TRI. ${ }^{24}$ Significantly increased proportions of GSTT1 present and GSTM1 present individuals were found among 45 RCC cases with a history of long term exposure to TRI, compared to 48 similarly exposed subjects not suffering from any cancer. ${ }^{17}$ A significant excess of transversions in the VHL gene has been recently found in a subset of sporadic RCC cases bearing a GSTTl present genotype. $^{25}$ Our results agree with these data, clearly indicating a strong interference of the GSTT1 present genotype on the risk of developing RCC among subjects exposed to organic solvents. GSTT1-1 has a substrate specificity for halogenated solvents, which are generally bioactivated in the kidney after GSH conjugation. When the same exposed subjects were classified for GSTM1 polymorphism, both GSTMI present and GSTMI null genotypes were significantly associated with RCC, showing an effect of exposure rather than a modifying role of the polymorphism. With regard to metal exposure, a significant association was found among subjects with the GSTTl present genotype or a GSTT1 present and GSTMl present combination. The GSTM1 present genotype also enhanced the risk, but, in our data, the association failed to reach full statistical significance. In animal models, exposure to metals (that is, mercury and lead) is known to induce GST expression in both liver and kidney. ${ }^{26}{ }^{27}$ Thus, a metabolic induction of GST activity, with subsequent increased production of potentially nephrotoxic glutathione conjugated compounds, could be envisaged among GSTM1 present and GSTT1 present subjects occupationally exposed to metals. Organic solvents are extensively used in the metal industries, however, which could imply some uncontrolled confounding in this respect ( 10 cases and 17 controls have been classified as exposed to both solvents and metals). The number of subjects with a single exposure is too small to permit further analysis.

Pesticides represent a wide class of compounds that change over time, making retrospective analyses difficult. Their carcinogenic potential is of greatest concern as a long term effect of pesticide exposure. ${ }^{28}$ Some suggestions of a possible relation between GST status and early markers of genotoxic effects in humans exposed to pesticides are available. An increased frequency of micronuclei in cultured peripheral lymphocytes has been found among pesticide exposed greenhouse workers with the GSTMI present genotype. ${ }^{29}$ Significantly higher levels of sister chromatid exchanges were also found among GSTT1 present individuals exposed to pesticides when compared to GSTT1 null workers similarly exposed. ${ }^{30}$ These results are consistent with the increased risk for RCC found here for both GSTMI present and GSTTI present genotypes, considered either separately or in combination. Some pesticides are produced from halogenated alkanes, and alkenes of low molecular weight, in a number of chemical processes; hence, a similar pathway of nephrotoxicity may be hypothesised as that proposed for halogenated solvents.

The main limitation of the present study is its small sample size, limiting the statistical power for many of the possible gene-environmental interactions. A power higher than $80 \%$ was achieved for interactions between GSTMI present genotype for metals as well as pesticides. However, several 
thousand cases and controls would be required to detect significant gene-environmental or gene-gene-environmental interactions for polymorphism showing a prevalence lower than $50 \%$ (in this case, the GSTT1 polymorphism). Another limitation is represented by broad exposure categories resulting from exposure assessment based on questionnaires, which represent a relatively poor tool to identify specific agents and to estimate exposure levels in the workplace. Nevertheless, the good agreement between our results and biological models of nephrocarcinogenicity makes our findings interesting and exciting.

In conclusion, broad exposure categories result from exposure assessment relying on questionnaires, which represent a relatively poor tool to identify specific agents and to estimate exposure levels in the workplace. The present study has shown that biologically plausible interactions between genetic traits and environmental factors may play a role in the development of RCC. Previous epidemiological studies on RCC, investigating the role of genetic polymorphisms as risk factors, gave rise to weak and inconsistent findings,${ }^{15}{ }^{16}$ but polymorphic enzymes are determinants of susceptibility and not of disease. They are expected to contribute to the pathogenesis of multifactorial diseases by modulating the effects of relevant exogenous risk factors. In view of its limited sample size, the present investigation on gene-environment interaction in RCC requires confirmation by much larger studies.

\section{ACKNOWLEDGEMENTS}

Supported in part by the Italian Ministry of Health through a grant from ISPESL (Istituto Superiore per la Prevenzione e la Sicurezza del Lavoro, Rome, Italy).

\section{Authors' affiliations}

L Buzio, G De Palma, P Mozzoni, C Buzio, I Franchini, A Mutti, Dept of Clinical Medicine, Nephrology and Health Sciences, University of Parma, Parma, Italy

M Tondel, O Axelson, Division of Occupational and Environmental Medicine; Department of Molecular and Clinical Medicine, Faculty of Health Sciences, University Hospital, Linköping, Sweden

\section{REFERENCES}

1 La Vecchia C, Negri E, Levi F. Increasing incidence of renal cell cancer. JAMA 1999;282:2120-1.

2 Chow WH, Devesa S, Warren J, et al. Rising incidence of renal cell cancer in the United States. JAMA 1999;281:1628-31.

3 McLaughlin JK, Chow WH, Mandel JS, et al. International renal-cell cancer study. VIII.Role of diuretics, other anti-hypertensive medications and hypertension. Int J Cancer 1995;63:216-21.

4 McCredie M, Stewart JH. Risk factors for kidney cancer in New South Wales. I.Cigarette smoking. Eur J Cancer 1992;28:2050-4.

5 Chow WH, Gridley G, Fraumeni J, et al. Obesity, hypertension and the risk of kidney cancer in men. N Engl J Med 2000;343:1305-11.

6 Pesch B, Hearting J, Ranft U, et al. Occupational risk factors for renal cell carcinoma: agent-specific results from a case-control study in Germany. Int J Epidemiol 2000;29:1014-24.
7 Parent ME, Hua Y, Siemiatycki J. Occupational risk factors for renal cell carcinoma in Montreal. Am J Ind Med 2000;38:609-18.

8 Mellemgaard A, Engholm G, McLaughlin JK, et al. Occupational risk factors for renal-cell carcinoma in Denmark. Scand J Work Environ Health 1994;20:160-5.

9 Meyer AJ, Hernandez A, Florl AR, et al. Novel mutations of the Von HippelLindau tumor-suppressor gene and rare DNA hypermethylation in renal-cell carcinoma cell lines of the clear-cell type. Int J Cancer 2000;87:650-3.

10 Bruning T, Weirich G, Huornauer MA, et al. Renal cell carcinomas in trichloroethene (TRI) exposed persons are associated with somatic mutations in the Von Hippel-Lindau (VHL) tumour suppressor gene. Arch Toxicol 1997;71:332-5.

11 lliopoulos O, Eng C. Genetic and clinical aspects of familial renal neoplasms. Semin Oncol 2000;27:138-49.

12 Hewitt SM, Hamada S, McDonnell TJ, et al. Regulation of the proto-oncogenes bcl-2 and c-myc by the Wilms' tumor suppressor gene WT1. Cancer Res 1995;55:5386-9.

13 Landi S. Mammalian class theta GST and differential susceptibility to carcinogens: a review. Mutat Res 2000;463:247-83.

14 Garte S, Gaspari L, Alexandrie AK, et al. Metabolic gene polymorphism frequencies in control populations. Cancer Epidemiol Biomarkers Prev 2001;10:1239-48.

15 Longuemaux S, Delomenie C, Gallou C, et al. Candidate genetic modifiers of individual susceptibility to renal cell carcinoma: a study of polymorfic human xenobiotic-metabolizing enzymes. Cancer Res 1999;59:2903-8.

16 Sweeney C, Farrow DC, Schwartz SM, et al. Glutathione S-transferase M1, $\mathrm{Tl}$, and $\mathrm{Pl}$ polymorphisms as risk factors for renal cell carcinoma: a casecontrol study. Cancer Epidemiol Biomarkers Prev 2000;9:449-54.

17 Brüning T, Lammert M, Kempkes M, et al. Influence of polymorphisms of GSTM1 and GSTT 1 for risk of renal cell cancer in workers with long-term high occupational exposure to trichloroethene. Arch Toxicol 1997;71:596-9.

18 Arand M, Muhlbauer R, Hengstler J, et al. A multiplex polymerase chain reaction protocol for the simultaneous analysis of the glutathione S-transferase GSTM1 and GSTT1 polymorphisms. Anal Biochem 1996;236:184-6.

19 Breslow NE, Day NE. Statistical methods in cancer research. Vol. I. The analysis of case-control studies. Sci Publ No 32. Lyon, France: IARC, 1980.

20 Gauderman WJ. Sample size requirements for matched case-control studies of gene-environment interaction. Am J Epidemiol 2002;5:478-84.

21 Buzio L, Tondel M, De Palma G, et al. Occupational risk factors for renal cell cancer. An Italian case-control study. Med Lav 2002;93:303-9.

22 Groves CE, Lock EA, Schnellmann RG. Role of lipid peroxidation in renal proximal tubule cell death induced by haloalkene cysteine conjugates. Toxicol Appl Pharmacol 1991;107:54-62.

23 Dekant W, Vamvakas S, Berthold K, et al. Bacterial beta-lyase mediated cleavage and mutagenicity of cysteine conjugates derived from the nephrocarcinogenic alkenes trichloroethylene, tetrachloroethylene and hexachlorobutadiene. Chem Biol Interact 1986;60:31-45.

24 Henschler D, Vamvakas S, Lammert $M$, et al. Increased incidence of renal cell tumors in a cohort of cardboard workers exposed to trichloroethene. Arch Toxicol 1995;69:291-9.

25 Gallou C, Longuemaux S, Delomenie C, et al. Association of GSTT1 non-null and NAT1 slow/rapid genotypes with von Hippel-Lindau tumour suppressor gene transversions in sporadic renal cell carcinoma. Pharmacogenetics 2001;11:521-35.

26 Witzmann FA, Daggett DA, Fultz CD, et al. Glutathione S-transferases: twodimensional electrophoretic protein markers of lead exposure. Electrophoresis 1998;19:1332-5.

27 McGuire S, Daggett DA, Bostad E, et al. Increased levels of glutathione S transferases and appearance of novel alpha class isoenzymes in kidneys of mice exposed to mercuric chloride. I.Biochemical and immunohistochemical studies. Nephron 1997;77:452-60.

28 Infante-Rivard C, Labuda D, Krajinovic $M$, et al. Risk of childhood leukemia associated with exposure to pesticides and with gene polymorphisms. Epidemiology 1999; 10:481-7.

29 Falck GC, Hirvonen A, Scarpato R, et al. Micronuclei in blood lymphocytes and genetic polymorphism for GSTM1, GSTT1 and NAT2 in pesticideexposed greenhouse workers. Mutat Res 1999;441:225-37.

30 Scarpato R, Migliore L, Hirvonen A, et al. Cytogenetic monitoring of occupational exposure to pesticides: characterization of GSTM1, GSTT1, and NAT2 genotypes. Environ Mol Mutagen 1996;27:263-9. 